\title{
Optimized Face Recognition Algorithm using Spatial and Transform Domain Techniques
}

\author{
Sujatha $\mathrm{BM}^{1}$, K Suresh Babu ${ }^{2}$, Raja $\mathrm{K} \mathrm{B}^{2}$ and Venugopal K R \\ ${ }^{1}$ Department of Electronics and Communication Engineering, Acharya Institute of Technology, Bangalore, India. \\ ${ }^{2}$ Department of Electronics and Communication Engineering, University Visvesvaraya College of Engineering, Bangalore, India. \\ ${ }^{3}$ University Visvesvaraya College of Engineering, Bangalore, India. \\ ${ }^{1}$ sujathabm2005@gmail.com, 22ksb1559@gmail.com,2raja_kb@yahoo.com
}

\begin{abstract}
The biometrics is used to identify or verify persons effectively in the real time scenario. In this paper, we propose Optimized Face Recognition Algorithm using Spatial and Transform Domain Techniques. The face images are preprocessed using Discrete Wavelet Transform (DWT), resize and filtering. The Compound Local Binary Pattern (CLBP) is used to generate magnitude and sign components from preprocessed face images. The histogram is applied on sign and magnitude components of CLBP to compress number of features. The generated histogram features are concatenated to form CLBP-Histogram features. The Fast Fourier Transformation (FFT) is applied on preprocessed image and FFT magnitude features are generated. The CLBP-Histogram features are fused with FFT magnitude features to generate final feature set. The final feature sets of test image and data base images are compared using Euclidian Distance (ED) to recognise a person. It is observed that the performance parameter of the proposed algorithm is better compared to existing algorithms.
\end{abstract}

Keywords- Biometrics; Face recognition; DWT; FFT; CLBP; Histogram.

\section{INTRODUCTION}

The biometrics stands for life measurements i.e., the measurement and analysis of biological characteristics such as fingerprints, iris patterns, retina etc. or the behavioural characteristics such voice, gait, signature etc. of an individual. The identification of a person is based on biological factors and behavioural characteristics. Some of the different types of biological biometrics are: (i) Fingerprint Recognition: The ridges and valleys in the fingertips to identify an individual. The drawback with this approach is that most often it is possible for an individual to lose the fingerprints due to injury or due to working in hazardous work environments. (ii)Face Recognition: The facial characteristics of an individual are analysed. (iii)Finger/Hand Geometry Recognition: The special and transform domain features are analysed for recognition.(iv)Iris Recognition: An individual is identified based on the unique patterns of the iris. (v) Retina Recognition: Analysing the features of the capillary vessels present at the back of the eye.(vi)Vein Detection: The vein patterns in the back of the hand and the wrist to identify an individual. The analysis of various behavioural characteristics to identify an individual are: (i) Voice Recognition: Analysing the tone, frequency and pitch of an individual's voice. (ii)
Signature Recognition:Analysis of the style in which a person does thesignature. (iii) Keystroke Recognition: Studying the pattern in which an individual types on the keyboard.

Face recognition is the biometric technique of identifying through the analysis of facial features. The key advantage of face recognition is that it does not require any cooperation from the subject under test. Most face recognition systems implemented in surveillance applications captures and analyses the individuals even without their knowledge. Face recognition has extensive application in both one-to-one mapping for verification of a person and one-to-many mapping for identification of an individual. One of the ways to achieve this is by first acquiring the facial features and then comparing it with the facial databases. The advantages and applications of face recognition is a hot field of research and challenging task for efficient identification under illuminations pose variation etc.

Contribution: In this paper Optimized Face Recognition Algorithm using Spatial and Transform Domain Techniques is proposed. The Face images are preprocessed using DWT. The CLBP features are generated from preprocessed face images. The FFT is applied on face images to generate FFT magnitude features. The CLBP and Histogram features are fused with FFT magnitude features to generate final features.

Organisation:The remaining sections of this paper are structured as follows. Section II explains the literature survey of existing techniques. Section III describes the proposed face recognition model. The proposed algorithm is given in section IV. The performance analysis is discussed in section V.Conclusion is given Section VI.

\section{LITERATURE SURVEY}

Faisal R.AI-Osaimi et al., [1] proposed a spatially optimised data/pixel-level fusion of 3D shape and texture. Here in order to make the expression and illumination variations reside better in Principal component analyses (PCA) subspace, the fusion is spatially optimised with respect to multimodal pixel values. Also they have proved that identification performance was further improved by using higher order fusion functions and multiple fusion functions systematically.RaghuramanGopalan et al., [2] proposed blur robost descriptor for face recognition. The subspace is 
generated from convolution of an image with blur kernel of an image. This technique is used to recognise face images from blurred face images. Ping-Han Lee et al., [3] explored the orientations of edges and proposed Oriented Local Histogram Equalization (OLHE) which compensates illumination. The OLHE feature combination schemes are used viz, encoded most edge orientations, compactness with good edgepreserving capabilities and perform better in extreme lighting conditions. Discriminant nonlinear with Generalise Discriminant Analysis (GDA) was applied to LBP, Gabor and Local TernityPatterns (LTP) are used for feature extraction. Cosine distance based nearest neighbour is used for matching.Gae Yong Choi et al.,[4] proposed color texture features based on Color Local Gabor Wavelets (CLGWs) andColor Local Binary Pattern (CLBP) for face recognition. To perform classification multiple color local textures are combined at the feature level with uniform weights.

\section{PROPOSED MODEL}

The proposed model is shown in Fig 1. The face images are preprocessed using DWT, Laplacian filtering and subtraction for better performance

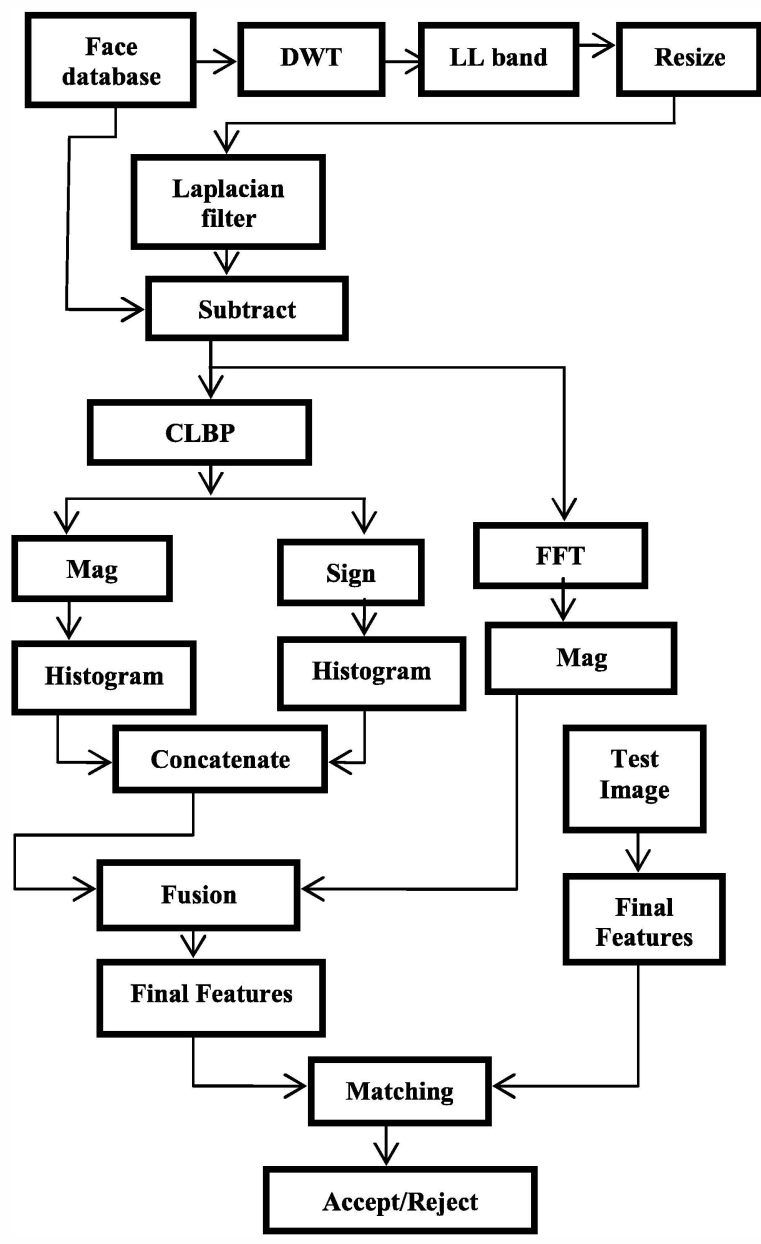

Fig 1: Block diagram of proposed model

\section{A. Face Database}

The standard face databases such as JAFFE and ORL are considered to test the proposed model.

\section{B. Discrete Wavelet Transform}

The Two Dimensional-Discrete Wavelet Transform (2DDWT) is a multilevel decomposition technique that converts the images from spatial to transform domain[5].One level wavelet decomposition produce four sub bands such as one approximation band and three detailed bands. 2D-DWT is implemented as a convolution of mother wavelet function with the original image or it can be viewed as a set of two matrices of filters with row and columns. The mathematical representation of decomposition is given as equation (1)

$$
\mathrm{C}_{\mathrm{f}}=\mathrm{X} \times \mathrm{I} \times \mathrm{Y}
$$

Where $\mathrm{C}_{\mathrm{f}}$ is the final matrix of wavelet coefficients, $\mathrm{I}$ is the original image.

$\mathrm{X}$ is elements of row filters.

$\mathrm{Y}$ is elements of column filters.

The input image in each level is split into four bands using the low pass and high pass wavelet filters on the rows and columns. The row filters in each level are used to convert an image into low and high frequency components. The column filters in each level are used to convert low frequency components of row filters into low and high frequency components. Similarly high frequency components of row filters are converted into low and high frequency components. In each level four sub bands are generated. The low frequency sub bands of level 1 act as input to the level 2 .

\section{Laplacian Filter}

It is used for image edge detection. The laplacian filter is applied on resized LL band coefficients of DWT. The original image coefficients are subtracted from LaplacianFilter coefficients to generate sharpened image.

\section{Compound Local Binary Pattern}

It is an extension of the Local Binary Pattern (LBP) texture operator. The image is converted into number of cells of dimension $3 * 3$. The centre pixel in the $3 * 3$ cell is considered as reference to generate Compound Local Binary Pattern (CLBP) texture operator with LSB for magnitude component and MSB for sign component. Theintensity value of centre pixel is say Ic and intensity values of surrounded pixels are represented by Ip.

The sign component of CLBP is generated using equation (2)

CLBP_Sign $= \begin{cases}0: & I p-I c \leq 0 \\ 1: & I p-I c>0\end{cases}$

The magnitude component of CLBP is generated using equation (3) 


$$
C L B P_{-} M a g=\left\{\begin{array}{l}
0: I p-I c \leq M_{\text {avg }} \\
1: I p-I c>M_{\text {avg }}
\end{array}\right.
$$

Where, $\mathrm{M}_{\mathrm{avg}}=(|\mathrm{m} 1|+|\mathrm{m} 2|$. $+|\operatorname{m} 8|) / 8$

$\mathrm{m} 1$ to $\mathrm{m} 8$ are the magnitude values of difference between respective Ipand $I c$.

\section{E. Histogram}

Histogram or specifically image histogram is the representation of an image in terms of a bar graph of pixel intensities. Histogram is a powerful tool used to view the image contrast and intensity distribution [6]. The histogram for image consists of pixel intensities plotted along the $\mathrm{x}$-axis and the frequency of these pixel intensities along the $y$-axis. The histogram reduces number of features, which is advantage in real time application.

\section{F. Fast Fourier Transform (FFT)}

The tool used to decompose an image into cosine (real) and sine (imaginary) components. The transformation is the representation of input image into frequency domain. FFT algorithm is based on either divide and conquers method or linear filtering. The divide and conquer method includes FFT algorithms like Radix-2 and Radix-4 uses Decimation in Time (DIT) and Decimation in Frequency (DIF). FFT is facilitates computation of Discrete Fourier Transform (DFT) in real time which reduces the complexity and reduces the computational time. The FFT and its inverse of 2D image can be computed using the equations no's (4) and (5).

DFT: $\mathbf{F}(\mathbf{x}, \mathbf{y})=\sum_{m=0}^{M-1} \sum_{n=0}^{N-1} f(m, n) e^{-j 2 \pi\left(x \frac{m}{M}+y \frac{n}{N}\right)}$

IDFT:f (m, n) $=\frac{1}{M N} \sum_{m=0}^{M-1} \sum_{n=0}^{N-1} F(x, y) e^{j 2 \pi\left(x \frac{m}{M}+y \frac{n}{N}\right)}$

Where, $M$ is the number of rows.

$N$ is the number of columns.

It is observed from the equations (4) and (5) that the implementation is complex and expensive. Hence the 2D transform is split as two 1D transforms, one in horizontal direction and the other in the vertical direction. The end result is equivalent to $2 \mathrm{D}$ transform in frequency domain. The 1-D DFT and IDFT are given in equations (6) and (7).

$$
\begin{aligned}
& \mathrm{F}(\mathrm{x})=\sum_{n=0}^{N-1} f(n) e^{-j 2 \pi\left(x \frac{n}{N}\right)} \\
& \mathrm{f}(\mathrm{n})=\frac{1}{N} \sum_{n=0}^{N-1} F(x) e^{j 2 \pi\left(x \frac{n}{N}\right)}
\end{aligned}
$$

The magnitude values of $F(X)$ are very high compared to pixel intensity values in the spatial domain and features are represented by $\mathrm{D}$.

\section{G. Fusion}

The CLBP-Histogram features and FFT magnitudes are fused using equation (8)to generate finalfeatures of an image.

FinalScore $F=P * D+(1-P) * C$
Where $P$ is improved factor varies from 0 to 1

$\mathrm{D}$ is transform domain features

$\mathrm{C}$ is CLBP- histogram features

\section{H. Euclidian Distance}

The distance between test and database features is measured using Euclidian distance (ED) to identify a person using equation (9).

$$
\mathrm{E} \mathrm{D}=\sqrt{\sum_{i=1}^{M}(\mathrm{Pi}-\mathrm{qi}) 2}
$$

Where, $M=$ Total number of feature coefficients.

$\mathrm{Pi}=$ Feature Coefficient values of database.

$\mathrm{Q} i=$ Feature Coefficient values of test image.

\section{ALGORITHM}

Problem Definition: The hybrid domain technique is used for face recognition with DWT in the Preprocessing.

The objectives are

i) To maximiseTotal Success Rate (TSR)

ii) To minimise False Rejection Rate (FRR),False Acceptance Rate (FAR) and Equal Error Rate (EER)

The proposed face recognition algorithm using DWT, Filtering, CLBP, Histogram and FFT is given in Table 1

TABLE 1: PROPOSED FACE RECOGNITION ALGORITHM

Input: Face Images

Output: Performance parameters

1. The Face Images are pre-processed using DWT, resize, filtering and subtract.

2. The CLBP is used on preprocessed face images to compute magnitude and sign components.

3. The Histogram is applied on magnitude and sign components of CLBP and concatenate the Histograms of magnitude and sign.

4. The FFT is applied on preprocessed image to generate FFT magnitude features.

5. The FFT magnitude features are fused with concatenated histogram features to generate final features.

6. The final set of features of test and data base images are compared using Euclidian Distance.

7. Compute performance parameters such as TSR, FRR, FAR and EER. 


\section{PERFORMANCE ANALYSIS}

The performance analysis of proposed work is discussed in this section using performance parameters viz., FRR, FAR, EER and TSR for different face databases.

\section{A. Analysis using JAFFE Database}

\section{(i) Proposed Hybrid Technique}

The database is created to test the performance of an algorithm by considering eight persons inside database and two persons outside database. The variations of FAR, FRR and TSR with threshold using CLBP technique with/ without Preprocessing are shown in Figure 2. The values of FRR decrease with increase in threshold values. The FAR and TSR values increase with increase in threshold values. The Maximum TSR (Max.TSR) obtained without and with preprocessing face images are $88.33 \%$ and $100 \%$ respectively. The EER values are less with preprocessing compared to without preprocessing.

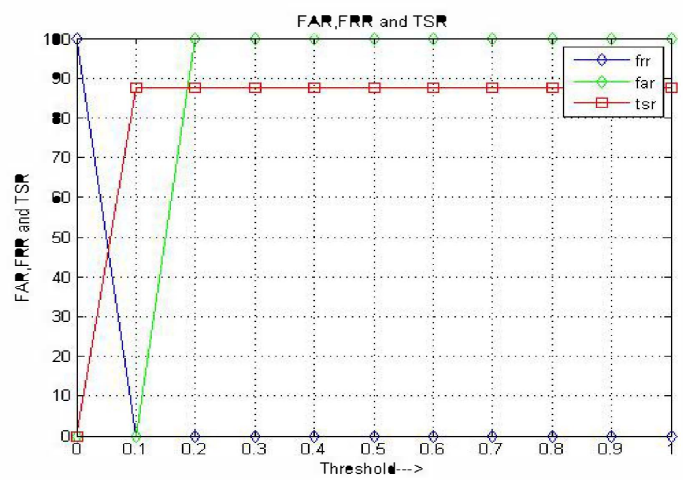

(a) Without Preprocessing

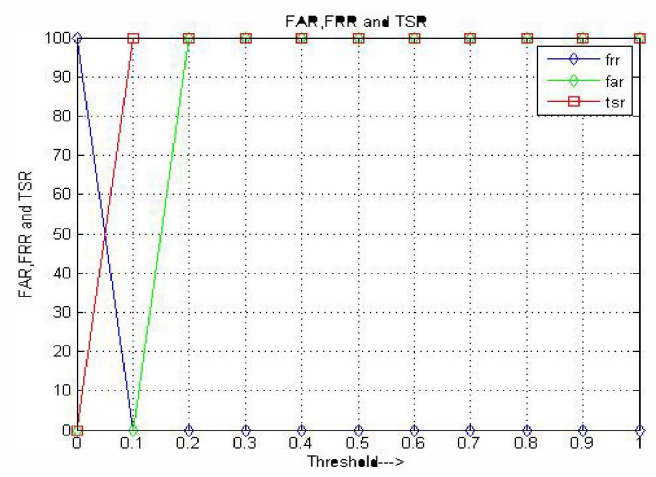

(b) With Preprocessing

Fig 2: The performance parameter variations with threshold for hybrid technique

(ii) Performance comparison of CLBP, FFT and Hybrid Techniques

The performance parameters viz., EER, Optimum TSR (Opt,TSR) and Maximum TSR (Max.TSR) for CLBP, FFT and Hybrid domain techniques are tabulated in Table 2. The values of EER with Preprocessing are lower compared to without Preprocessing. In all the three techniques the values of Max.TSR and Opt.TSR are higher in the case of with Preprocessing compared to without preprocessing. It is observed that the values EER are zero in the case proposed hybrid technique compared to CLBP and FFT techniques. The values Opt. and Max. TSR with and without Preprocessing are high in the case of hybrid technique compared to CLBP and FFT techniques.

TABLE 2: Percentage VARIATIONS Of FAR, FRR AND TSR WITHOUT AND WITH PREPROCESSING

\begin{tabular}{|c|c|c|c|}
\hline \multicolumn{2}{|c|}{} & $\begin{array}{c}\text { Without } \\
\text { preproces } \\
\text { sing }\end{array}$ & $\begin{array}{c}\text { With } \\
\text { preprocessi } \\
\text { ng }\end{array}$ \\
\hline \multirow{4}{*}{ CLBP } & EER (\%) & 40 & 36 \\
\cline { 2 - 4 } & Opt.TSR (\%) & 50 & 55 \\
\cline { 2 - 4 } & Max.TSR(\%) & 75 & 87.5 \\
\hline \multirow{4}{*}{ FFT } & EER (\%) & 20 & 12 \\
\cline { 2 - 4 } & Opt.TSR (\%) & 80 & 90 \\
\cline { 2 - 4 } Hybrid & Max.TSR(\%) & 100 & 100 \\
\hline \multirow{3}{*}{ Technique } & EER (\%) & 0 & 0 \\
\cline { 2 - 4 } & Opt.TSR (\%) & 88.33 & 100 \\
\cline { 2 - 4 } & Max.TSR(\%) & 88.33 & 100 \\
\hline
\end{tabular}

\section{B. Analysis using ORL Database}

\section{(i) CLBP Technique}

The database is created to test the performance of an algorithm by considering thirty persons insidedata base and ten persons outsidedata base. The variations of FAR, FRR and TSR with threshold using CLBP technique with / without Preprocessing are shown in Figure 3 . The values of FRR decreases with increase in threshold values. The FAR and TSR values increase with increase in threshold values. The Maximum TSR (Max.TSR) obtained without and with preprocessing face images are $73.33 \%$ and $83.33 \%$ respectively. The EER values are less with preprocessing compared to without preprocessing.

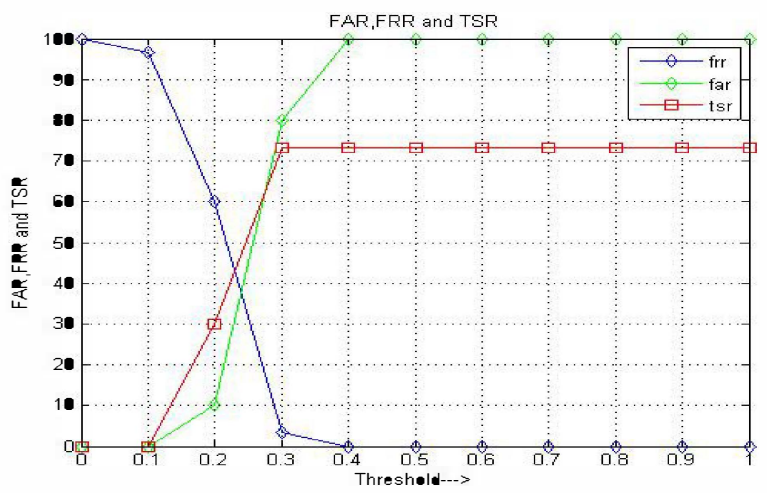

(a) Without Preprocessing 


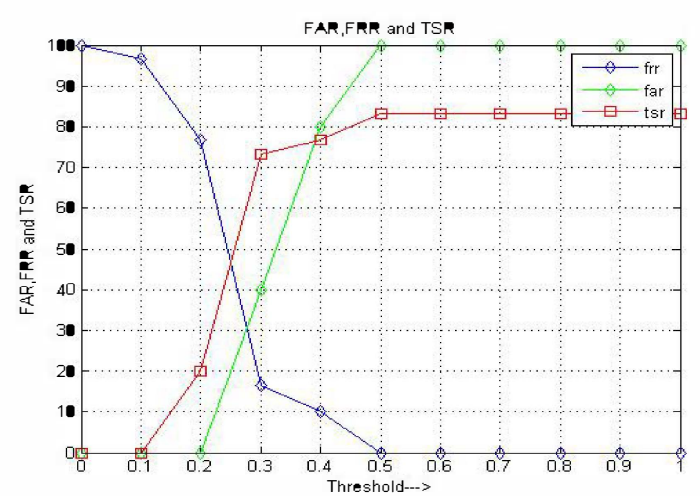

(b) With Preprocessing

Fig 3: The performance parameter variations with threshold for CLBP technique

\section{(ii) FFT Technique}

The variation of FAR, FRR and TSR with threshold using CLBP technique with / without Preprocessing is shown in Figure 4. The values of FRR decreases with increase in threshold values. The FAR and TSR values increase with increase in threshold values. The Maximum TSR (Max.TSR) obtained without and with preprocessing face images is $90 \%$. The EER values are less with preprocessing compared to without preprocessing.

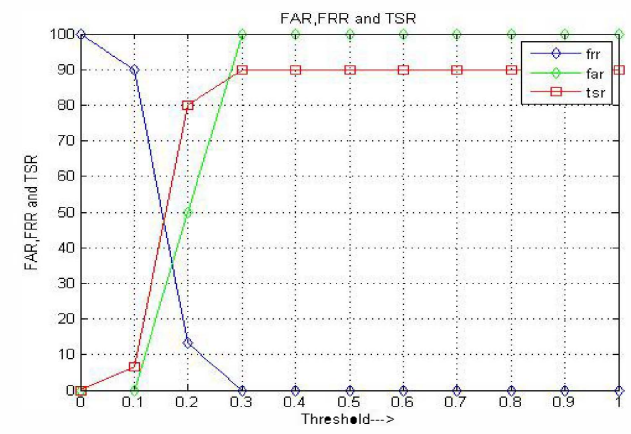

(a) Without Preprocessing

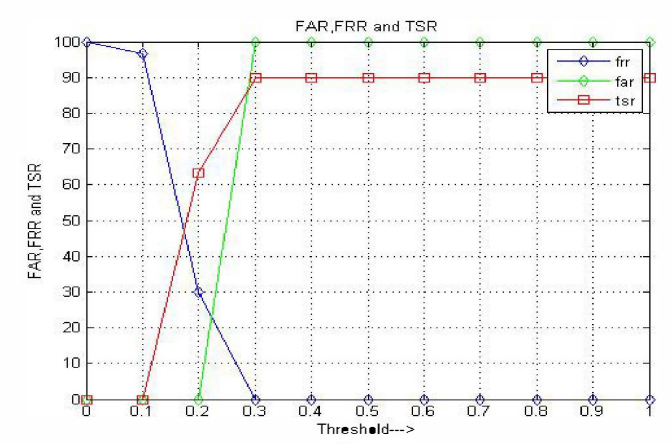

(b) With Preprocessing

Fig 4: The performance parameter variations with threshold for FFT technique

\section{iii) Proposed Hybrid Technique}

The variations of FAR, FRR and TSR with threshold using CLBP technique with / without Preprocessing are shown in Figure 5. The values of FRR decreases with increase in threshold values. The FAR and TSR values increase with increase in threshold values. The Maximum TSR (Max.TSR) obtained without and with preprocessing face images is 93.33 $\%$. The EER values are less with preprocessing compared to without preprocessing.

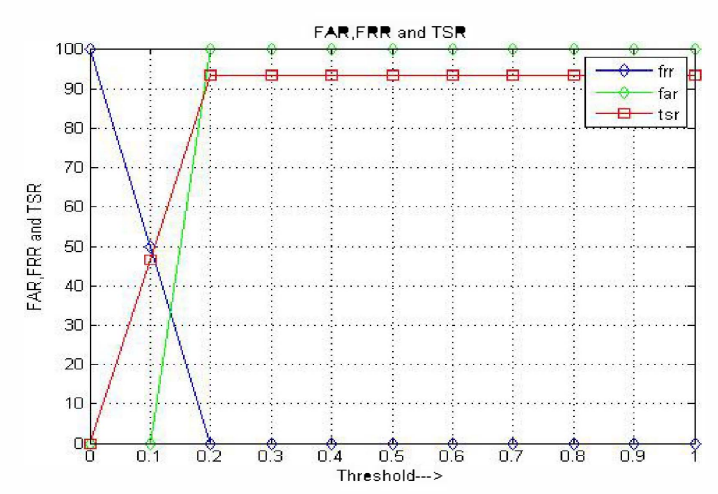

(a) Without Preprocessing

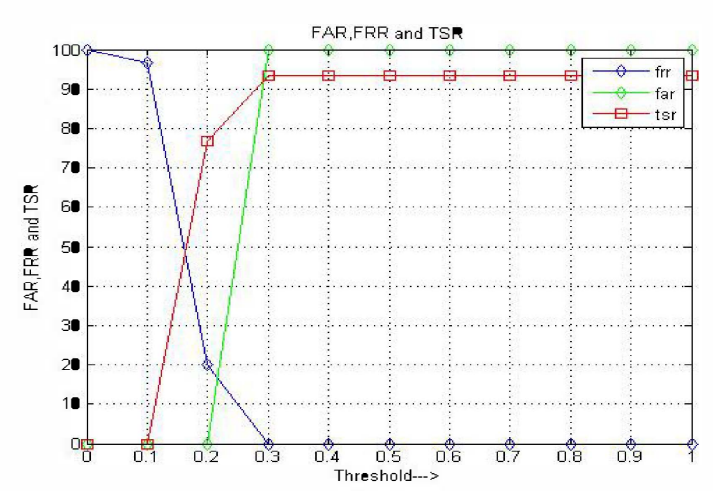

(b) With Preprocessing

Fig 5: The performance parameter variations with threshold for hybrid Technique

(iv) Performance comparison of CLBP, FFT and Hybrid Techniques

The performance parameters viz., EER, Optimum TSR and Maximum TSR for CLBP, FFT and Hybrid domain techniques are tabulated in Table 3 . The values of EER with preprocessing are lower compared to without preprocessing. In all the three techniques the values of Max.TSR and opt. TSR values are higher in the case of with preprocessing compared to without preprocessing. It is observed that the values EER are 32 and 18 without and with preprocessing respectively in the case proposed hybrid technique compared to CLBP and FFT techniques. The values Opt.TSR and 
Max.TSR with and without preprocessing are high in the case of hybrid technique compared to CLBP and FFT techniques.

TABLE 3: PERCENTAGE VARIATIONS OF FAR, FRR AND TSR wITHOUT AND WITH PREPROCESSING

\begin{tabular}{|c|c|c|c|}
\hline \multicolumn{2}{|c|}{ Techniques } & $\begin{array}{c}\text { Without } \\
\text { preprocessing }\end{array}$ & $\begin{array}{c}\text { With } \\
\text { preprocessing }\end{array}$ \\
\hline \multirow{4}{*}{ CLBP } & EER (\%) & 40 & 30 \\
\cline { 2 - 4 } & Opt.TSR (\%) & 45 & 60 \\
\cline { 2 - 4 } & Max.TSR (\%) & 73.33 & 83.33 \\
\hline \multirow{4}{*}{ FFT } & EER (\%) & 20 & 12 \\
\cline { 2 - 4 } & Opt.TSR (\%) & 60 & 68 \\
\cline { 2 - 4 } & Max.TSR (\%) & 90 & 90 \\
\hline \multirow{4}{*}{$\begin{array}{c}\text { Hybrid } \\
\text { Technique }\end{array}$} & Ept.TSR (\%) & 60 & 18 \\
\cline { 2 - 4 } & Max.TSR (\%) & 93.33 & 80 \\
\cline { 2 - 4 } & EER (\%) & 32 & 93.33 \\
\hline
\end{tabular}

\section{Performance Comparison for ORL database}

The percentage TSR of proposed algorithm for ORL database is compared with existing algorithm presented by Pallavi D. Wadakar and Megha Wankhade[7], Swarup Kumar Dandpat and Sukadev Meher[8], and D Murugan et al., [9] and is given in Table 4. It is observed that the percentage TSR is maximum in the case of proposed algorithm compared to existing techniques. The performance of proposed algorithm is better compared to existing algorithm for the following reason. (i) The DWT is applied on face images and only LL band is considered. The Laplacian filter is applied on resized LL band and manipulated with original face image to sharpen the face images. (ii) The CLBP magnitude and sign component produce texture feature which represents micro level information.(iii)The histogram compress number of CLBP features.(iv)The FFT magnitudes of laplacian filter coefficients are better features in frequency domain.(v)The spatial domain texture feature of CLBP are fused with frequency domain FFT feature to generate effective final feature set to identify a person properly.

TABLE 4: COMPARISON OF TSR WITH PROPOSED AND EXISTING ALGORITHMS

\begin{tabular}{|c|c|c|c|}
\hline $\begin{array}{c}\text { SI } \\
\text { No }\end{array}$ & Authors & Techniques & $\begin{array}{c}\text { TSR } \\
\%\end{array}$ \\
\hline 1 & $\begin{array}{c}\text { Pallavi D. Wadakar } \\
\text { and } \\
\text { MeghaWankhade[7] }\end{array}$ & DWT & 90 \\
\hline 2 & $\begin{array}{c}\text { Swarup Kumar } \\
\text { Dandpat and } \\
\text { SukadevMeher [8] }\end{array}$ & PCA+2DPCA & 90.5 \\
\hline 3 & D Murugan et al.,[9] & $\begin{array}{c}\text { Gaborfilter + } \\
\text { DWT + PCA }\end{array}$ & 92 \\
\hline 4 & Proposed Method & $\begin{array}{c}\text { DWT + } \\
\text { CLBP+FFT }\end{array}$ & $\mathbf{9 3 . 3 3}$ \\
\hline
\end{tabular}
"Spatially optimized Data-Level Fusion of Texture and Shape for Face Recognition," IEEE Transactions on Image Processing, Vol. 21, No.2, pp. 859-872, 2012

[2] Raghuraman Gopalan, SimaTaheri, Pavan Turaga, Rama Challappa, "A Blur-Robust Descriptor with Applications to Face Recognition," IEEE Transactions on Pattern Analysis and Machine Intelligence, Vol 34, No 6, pp. 1220-1226, 2012.

[3] Ping-Han Lee, Szu-Wei Wu and Yi-Ping Hung, "Illumination Compensation using Oriented Local Histogram Equalization and Its Application to Face Recognition," IEEE Transactions on Image processing, Vol 21, No 9, pp. 4280-4289, 2012.

[4] Jae Young Choi, Yong Man Ro and Konstantinos N. Plataniotis, "Color Local Texture Features for Color Face Recognition," IEEE Transactions on Image Processing, Vol. 21, No. 3, pp.1366-1380, 2012.

[5] J. Petrova, E. Ho-s talkova, "Edge Detection in Medical Images using the Wavelet Transform," Department of Computing and Control Engineering, Institute of Chemical Technology, Prague, Technicka 6, 16628 Prague 6, Czech Republic, 2011.

[6] Madhulakshmi, Abdul Wahid Ansari, "Face Recognition Using Featured Histogram," International Journal of Emerging Technology and Advanced Engineering, Vol. 3, Issue 8, pp.142- 147, 2013.

[7] Pallavi D.Wadkar and Megha Wankhade, "Face Recognition using Discrete Wavelet Transforms," International Journal of Advanced Engineering Technology, vol. 3, Issue 1, pp. 239-242, 2012.

[8] Swarup Kumar Dandapat and Sukadev Meher, "Performance Improvement for Face Recognition using PCA and Two-Dimensional PCA," International Conference on Computer Communication and Informatics, pp. 1-5, 2013.

[9] D Murugan, $\mathrm{S}$ Arumugam, $\mathrm{K}$ Rajalakshmi and Manish $\mathrm{T}$, "Performance Evaluation of Face Recognition using Gabor Filter, Log Gabor filter and Discrete Wavelet Transform," International Journal of computer science and Information Technology, vol. 2, no. 1, pp. 125 133,2010 . 